\title{
OSTEOARTHRITIS: A LOOK AT PATHOPHYSIOLOGY AND APPROACH TO NEW TREATMENTS: A REVIEW
}

S. H. Hassanali, MBBS, MMed, Consultant Physician, Aga Khan University Hospital, P. O. Box 30270, Nairobi, Kenya and G. O. Oyoo, MBChB, MMed, Cert Rhematology, FACR, FRCP (Edin), Consultant Physician and Rheumatologist, Senior Lecturer, Department of Clinical Medicine and Therapeutics, School of Medicine, University of Nairobi, P. O. Box 19676 - 00202, Nairobi, Kenya

Correspondence to: Dr. G. O. Oyoo, Department of Clinical Medicine and Therapeutics, School of Medicine, University of Nairobi, P. O. Box 19676 - 00202, Nairobi, Kenya. Email: goyoo@uonbi.ac.ke

\section{ABSTRACT}

Objective: To review old, current, emerging and potential pathophysiological mechanisms in osteoarthritis and its approach to new treatments.

Data sources: Published original research work and reviews from 1995 till early 2008 were searched in English on subjects related to pathogenesis, pathophysiology and potential new approaches to treatment of osteoarthritis.

Study design: Articles that emphasized the pathogenesis and pathophysiology and new treatment approaches to the management of osteoarthritis.

Data extraction: Online and manual library searches.

Data synthesis: Data added up and summarized.

Conclusion: Although osteoarthritis is characterized by cartilage degeneration, changes also involve the synovial membrane and the subchondral bone. Evidence suggests the involvement of a number of factors in the pathogenesis of osteoarthritis, like the proteases, pro-inflammatory cytokines, nitrous oxide, calcium crystals, leptin, angiogenesis and T cells and in turn using some of these to formulate some of the potential for new treatments for osteoarthritis.

\section{INTRODUCTION}

Osteoarthritis $(\mathrm{OA})$ is a group of chronic painful, disabling conditions affecting the synovial joints. It results from articular cartilage failure induced by a complex interplay of genetic, metabolic, biochemical, and biomedical factors with secondary components of inflammation. The process involves interactive degradation and repair processes of cartilage, bone and synovium. Osteoarthritis is the most common form of arthritis (1).

Among US adults 30 years of age and older, symptomatic disease in the knee occurs in approximately $6 \%$ and symptomatic hip osteoarthritis in roughly $3 \%$. Unfortunately we do not have figures to quote here in Kenya. Osteoarthritis is commonly described as a non inflammatory disease which distinguishes from inflammatory arthritis, such as rheumatoid arthritis (RA) or seronegative spondyloarthropathies. Despite this, inflammation is increasingly recognized as contributing to the symptoms and progression of OA. Chondrocytes are probably the most important cells responsible for the development of the osteoarthritic process. Human and animal studies indicate that chondrocytes exhibit numerous abnormal metabolic features as part of the osteoarthritic process. These include increased levels of proliferative $(2,3)$, synthetic $(4$, 5 ) and degradative activity. Monocytes -derived peptides may induce increased chondrocyte proliferative activity and matrix degradation. Other growth factors also stimulate chondrocyte synthesis. Cartilage fragments and soluble proteoglycan and type II collagen have been demonstrated in the synovial fluid of osteoarthritic joints. These cartilage degradative products can stimulate the release of inflammatory mediators from macrophages.

Two principle mechanisms are thought to initiate osteoarthritis (6): 
In most patients, the initiating mechanism is damage to normal articular cartilage by physical forces, which can be either single events of macrotrauma or repeated microtrauma. Chondrocytes react to this injury by releasing degradative enzymes and elaborating inadequate repair responses.

Less commonly, fundamentally defective cartilage initially fails under normal joint loading, thereby leading to osteoarthritis. Examples include a type Il collagen gene defect or ochronotic cartilage that fails because of deleterious pigment deposition.

The initiation of the osteoarthritic process therefore appears to involve abnormalities in biomechanical forces and/or cartilage. Once begun, the pathway leading to osteoarthritis involves numerous other factors. These include mechanotransduction, the interplay between proteases, protease inhibitors and cytokines on cartilage degradation and mechanisms of cartilage repair and the contributions from multiple risk factors (such as obesity, aging, mineral deposition, systemic hormones, and abnormalities in neurogenic control).

An abnormality associated with osteoarthritis extends beyond the cartilage often involving the subchondral bone, juxtaarticular bone marrow and the synovial membrane.

\section{PATHOPHYSIOLOGY OF OSTEOARTHRITIS}

A normal cartilage is avascular, alymphatic and aneural and is smooth and resilient in turn allowing shearing and compressive forces to be dissipated uniformly along a joint. The cartilage matrix is made up of water and gel (ground substance), which normally provides a "give", proteoglycans, and collagen.

Metallo proteases: A great deal of attention has been focused on identifying the protease responsible for the initial occurrence of matrix digestion. Current knowledge indicates an involvement of matrix metalloproteases (MMP). Of this family, members from three groups in human articular tissues have been identified as being elevated in OA that is the collagenases, the stromelysins and the gelatinases. Another group of MMP, localized at the cell membrane surface, has recently been discovered, and named membrane type MMP (MT-MMP), however the relevance of these enzymes to OA has yet to be determined. MMP biologic activity is controlled physiologically by specific tissue inhibitors of metallo-proteases (TIMP) or by their activation. Enzymes from the serine- and cysteine- dependent protease families, such as the plasminogen activation/plasmin system and cathepsin B respectively, has been proposed as activators, and enhanced levels of urokinase (UPA) and plasmin have been identified in human $O A$ cartilage. Other enzymes that have been found to act as MMP activators, for example stromelysin-1, activates collagenase- 1 , collagenase- 3 , and gelatinase $92 \mathrm{kD}$; collagenase- 3 activates gelatinase$92 \mathrm{kD}, \mathrm{MT}-\mathrm{MMP}$ activates collagenase-3 and gelatinase-72kD potentiates the later activation; MTMMP also activates gelatinase $72 \mathrm{kd}(7)$.

Inflammatory cytokines: Current evidence suggests that proinflammatory cytokines are responsible for the catabolic process occurring in the pathological tissues. They appear to be first produced by the synovial membrane, and diffused into the cartilage through the synovial fluid, where

Figure 1

The cartilage metabolism in OA whereby there is imbalance between the enzymes causing regeneration and degeneration

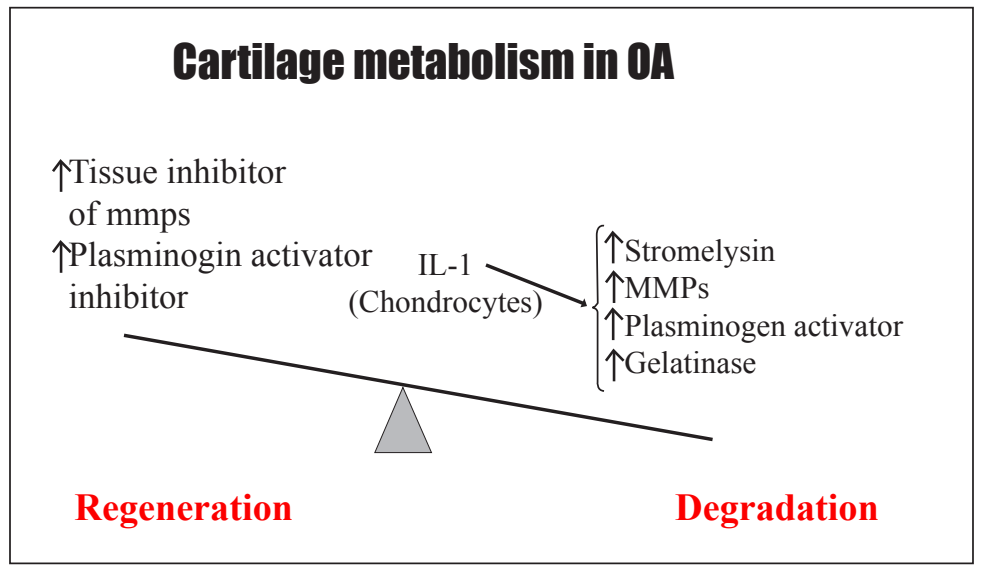


Figure 2

The cascade of pathology leading from the activation of pro inflammatory cytokines to the degradation of collagen within the cartilage

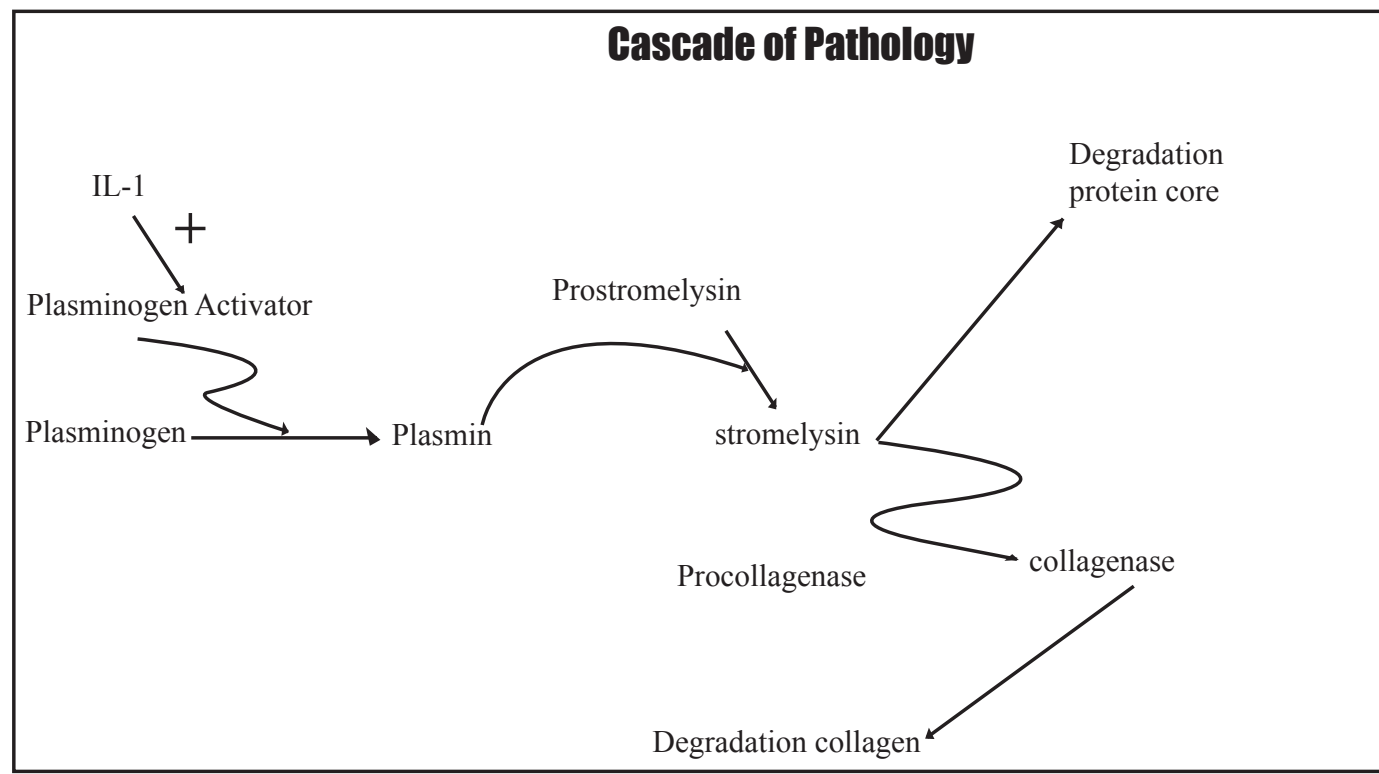

they activate the chondrocytes to produce proinflammatory cytokines. In OA synovial membrane, it is the synovial lining cells that play a major role as inflammatory effectors of which interleukin (IL)-1 $\beta$, tumor necrosis factor (TNF)- $\alpha$, IL-6, leukemic inhibitor factor (LIF) and IL-17 appear most relevant to the disease.

Data strongly suggests the concept that IL-1 $\beta$ and perhaps TNF- $\alpha$ are the major catabolic systems involved in the destruction of joint tissues, and may constitute the in situ source of articular tissue damage and degradation. However it is not clear whether IL-1 $\beta$ and TNF- $\alpha$ act independently or in concert to induce the pathogenesis of $O A$, or if a functional hierarchy exists between these proinflammatory cytokines. In animal models, it has been shown that blocking IL-1 or its activity is very effective in preventing cartilage destruction(8), whereas blocking TNF- $a$ results in decreased inflammation(9), however both cytokines have been found in enhanced amounts in OA synovial membrane, synovial fluid and cartilage. IL- 6 has been shown to be the contributor to OA pathological process by:

Figure 3

The involvement of the various cytokines in the process of osteoarthritis in turn affecting the quality of the cartilage matrix

\section{Cytokines in Osteoarthritis}

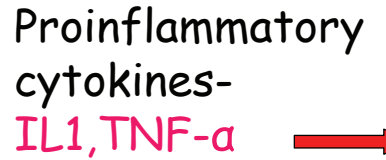

Regulatory cytokines, IL6,IL8,LIF

\section{Inhibitory cytokines}

\section{Initiation of disease process}
Quality of matrix is altered

$$
\begin{aligned}
& \text { - type } 1 \& 3 \text { collagen } \\
& \text { - } \text { type } 2 \& 9 \text { collagen }
\end{aligned}
$$

IL4,IL10,IL13,Interferon-y 
(i) Increasing the amount of inflammatory cells in the synovial tissue;

(ii) Stimulating the proliferation of chondrocytes; and

(iii) By inducing an amplification of IL-1 effects on the increased MMP synthesis and inhibition of proteoglycan production.

However IL-6 is involved in the feedback mechanism that limits enzyme production by also inducing the production of tissue inhibitor of matrix metalloproteinase's. LIF a cytokine of IL-6 family is also up regulated in OA synovial membrane and fluid and is produced by chondrocytes in response to proinflammatory cytokines which stimulates cartilage proteoglycan resorption, as well as MMP synthesis and NO cellular production; however its role in OA has not yet been clearly defined. IL-17 is a new cytokine that has been shown to up regulate a number of gene products involved in cell activation.

Nitrous Oxide: Inorganic free radical NO has also been suggested a factor that promotes cartilage catabolism in OA (10). A number of studies are on going to understand the molecular mechanisms underlying NO signaling and their effects on the cartilage.
Calcium Crystals: Calcium pyrophosphate has been shown to coexist with cartilage damage in some patients with OA, however what is not known as whether the crystals cause the cartilage damage or whether cartilage damage results in the formation of crystals. Calcium pyrophosphate has shown to be mitogenic; up regulate MMP's; stimulate the production of COX-1 and -2 and PGE2 production; stimulate cytokine production (IL) 10; and induce the production of NO production (11).

Leptins: Leptins have also been shown to play an important role in OA. There has been up regulation of leptin levels in synovial fluid, cartilage and osteophytes and its levels have been shown to correlate with the BMl; thus it is a key regulator of chondrocyte metabolism (12).

Angiogenesis: Recently angiogenesis and inflammation have been shown to play an important role in $\mathrm{OA}$ and may affect disease progression and pain. Angiogenesis and chronic inflammation are closely integrated processes. Inflammation can stimulate angiogenesis, and angiogenesis can in turn facilitate inflammation, including chondrocyte hypertrophy and endochondral ossification.

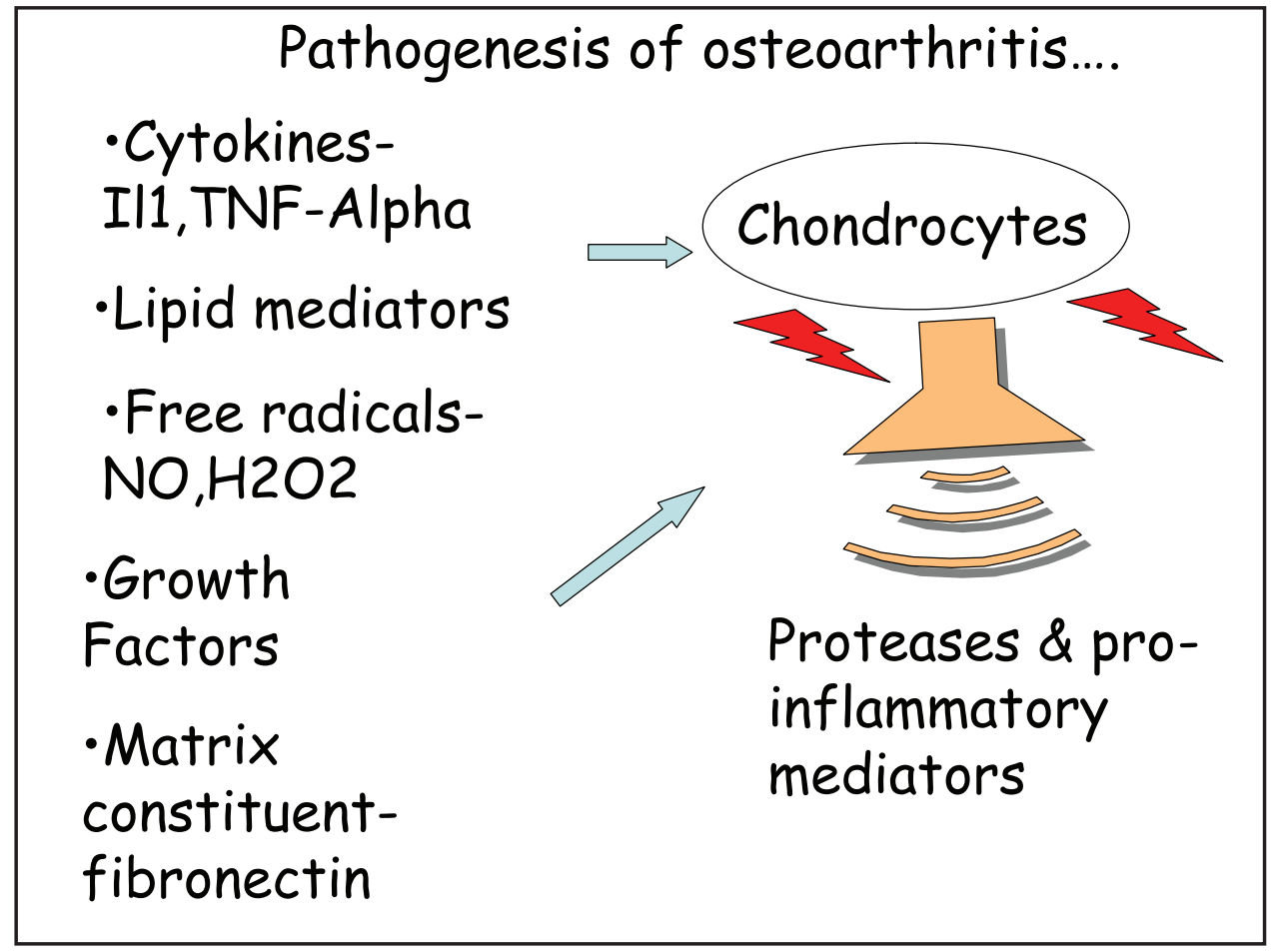


Inflammation may facilitate angiogenesis directly through the release of growth factors from cells such as macrophages, and also by stimulation or sensitization of other cells, such chondrocytes, nerves and osteoblasts, which in turn release additional angiogenic factors. Angiogenesis at the osteochondral junction leads to endochondral ossification and the formation of osteophytes. Angiogenesis and joint damage further exacerbate inflammation. New vessels, which breach the tidemark may later become innervated and could be a source of pain. Through these mechanisms, angiogenesis and inflammation can contribute pain and joint damage. Recent studies have implicated nitric oxide (NO) as a potential mediator of cartilage pathophysiology in osteoarthritis. NO exerts a major role in cartilage destruction and caused a pronounced suppression of proteogycan synthesis and IGF-1 unresponsiveness of the chondrocytes and also disrupts the cytoskeletal signaling complex associated wih integrin signaling. Unidentified genetic factors have also been implicated in the development of $\mathrm{OA}$ and a genetic component is supported by studies of families and twins. Clonal chromosomal aberrations, such as the gain of chromosomes 5 and 7, were observed in the synovial membrane of certain patients with OA. Alpha1-antitrypsin, a1- antichymotrypsin, gene polymorphisms and HLA alleles have been associated with generalized OA (13).

TCells: As noted earlier that inflammation in the synovial membrane of at least $50 \%$ of patients with
$O A$ is well documented. This inflammatory response exhibits features of a T cell immune response (14). Several lines of evidence support the view that $T$ cells may play an important role in the pathogenesis and progression of $\mathrm{OA}$, as follows:

(i) $\mathrm{CD} 3+\mathrm{T}$ cells infiltrate the synovial membrane of patients with $O A$

(ii) T cells infiltrating the synovial membrane of patients with OA express early, intermediate, and late activation antigens.

(iii) Association of HLA B8, HLA Cw4 with OA.

(iv) $\mathrm{T}$ cell cytokines are produced in the synovial membrane of patients with OA namely IL2, interferon $\gamma$, and IL10.

(v) Autoantibody responses in $\mathrm{OA}$, auto antibodies against specific target antigens were detected in patients with $O A$ as early as 20 years ago.

(vi) $\mathrm{T}$ cells infiltrating the synovial membrane of patients with $\mathrm{OA}$ contain oligonclonal populations of T lymphocytes.

(vii) Putative $O A$ antigens. Although the antigen or antigens that drive the immune response in $\mathrm{OA}$ have not been identified, possible candidates have been postulated like the autologous chondrocyte membranes and autologous fibroblasts membranes, but not to epithelial membranes.

(viii)Decreased expression of the $\mathrm{CD} 3 \zeta$ chain in $T$ cells infiltrating the synovial membrane of patients with $\mathrm{OA} . \mathrm{CD} \zeta$ is one of the $\mathrm{CD} 3$ proteins and plays a crucial role in the signal transduction pathway.

Figure 5

The role of T cells in the pathogenesis of osteoarthritis

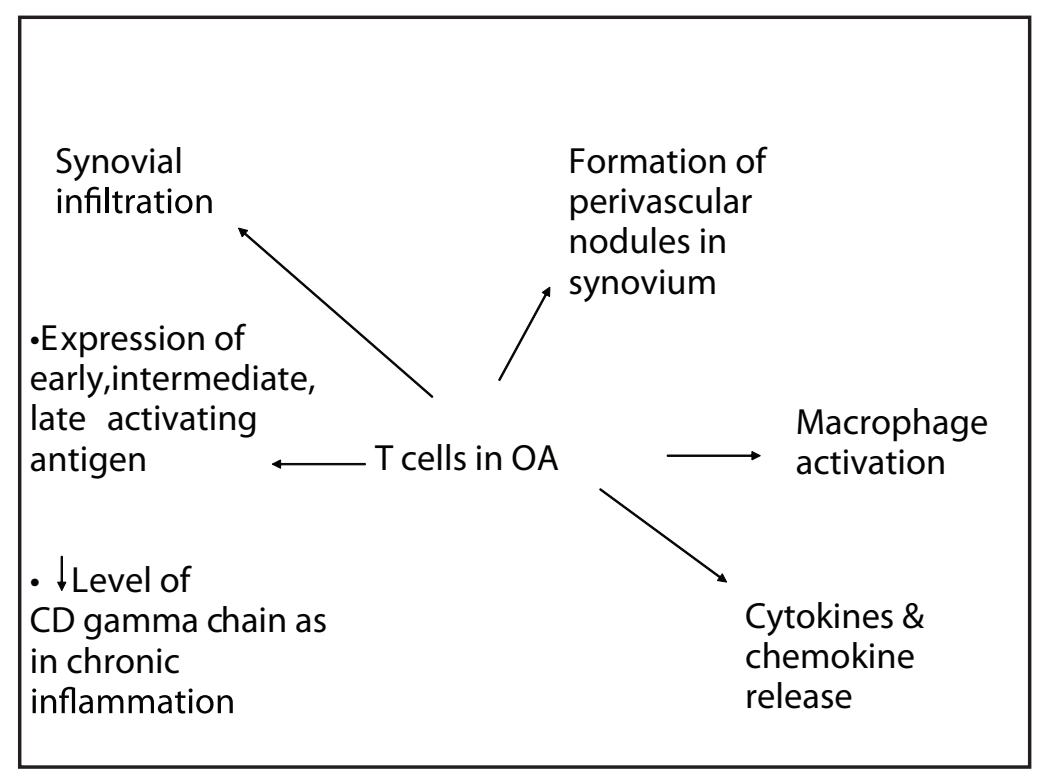


Figure 6

The involvement of the pro inflammatory cytokines and proposed sites for future treatment as shown by the negative -ve signs

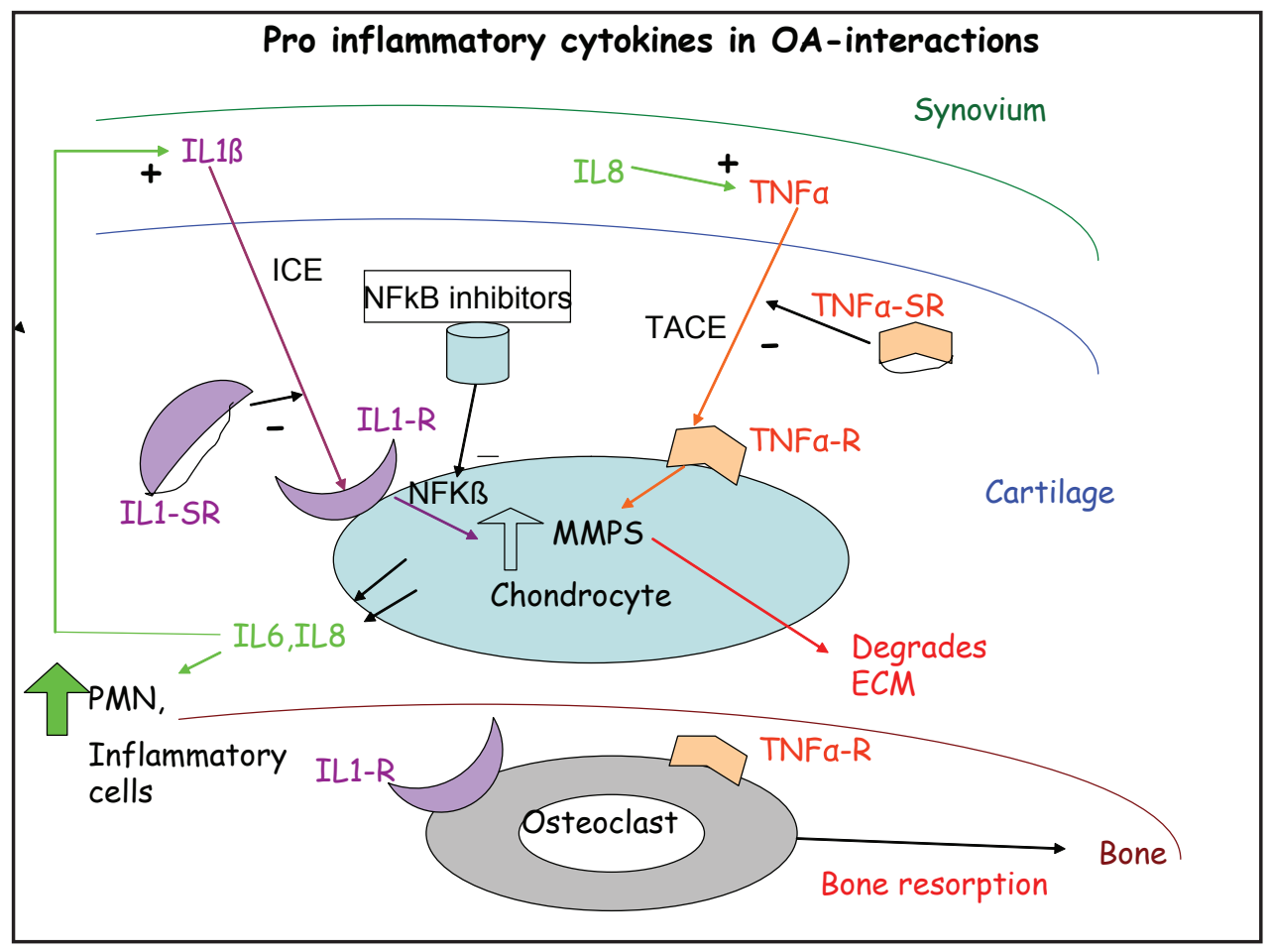

Although $O A$ is a heterogenous disease in many patients, a T cell immune response is evident and is characterized by the presence of T cell infiltrates, expression of T cell activation antigens, production of Th1 cytokines, and the presence of oligonal T cells in the synovial membrane.

\section{NEW THERAPEUTIC OPTIONS}

Natural inhibitors capable of directly counteracting the binding of the cytokine to the cells or reducing the proinflammatory level have been identified and have been divided into three categories based on their mode of action. The first inhibitor category is a receptor binding antagonist of which to date only the IL-1 receptor antagonist has been found. The second category is the soluble receptors for the IL$1 \beta$ and TNF- $\alpha$ that are the truncated forms of the receptors. Other natural inhibitors able to reduce pro inflammatory cytokine production and / or activity are the cytokines having anti inflammatory properties; namely transforming growth factor (TGF- $\beta$ ), IL-4, IL-10, and IL-13. Current therapeutics strategies of antagonizing IL-1 $\beta$ and TNF- $\alpha$ with either receptor blockade (with IL -1Ra) or molecular quenching (with IL-1R or TNF-R soluble receptors) have proven valuable in other arthritic diseases and in animal models of OA. Also in vitro effects of a selective inhibitor of iNOS on the progression of lesions in experimental arthritis and $O A$ have recently been reported (15). Other targets of therapy that have been looked at are the effect of tetracyclines in the inhibition of degradative enzyme proteases.

Neutraceuticals like glucosamine and chondroitin sulphate have been used in the modulation of cartilage constituents. Recently there has been a clinical review of chondroitin sulphate as an anti inflammatory agent (16). Others that have been tested with respect to their potential for disease modifying affects include diacerin, avocado and soya unsapponifiables,. Additional compounds including receptor activator of NF-қB (RANK) ligand inhibitors, cathepsin $\mathrm{K}$ inhibitors, bisphosphonates are further assessed regarding their potential structure modifying effects. Monoclonal antibodies like Etanercept for RA have been tried with little success although a number of clinical trials are ongoing and results awaited. 


\section{CONCLUSIONS}

It is now becoming clear that $\mathrm{OA}$ encompasses a broad spectrum of changes in the synovial membrane, ranging from minimal inflammation to intense inflammation, and that $O A$ may no longer be considered only a degenerative joint disease. Osteoarthritis is by far the most common type of arthritis encountered worldwide, yet the development of effective disease modifying treatments to lag behind that of the inflammatory arthritides. Current goals that still need to be achieved include the better understanding on how the various multi factorial processes manifest the osteoarthritis phenotype and improving early identification of patients with osteoarthritis both via clinical examination and biomarkers.

\section{REFERENCES}

1. Bonnet C. S and Walsh D. A.Osteoarthritis, angiogenesis and inflammation. Rheumatology. 2005;44:7-16.

2. Hulth, A, Lingberg, L. and Telhag, H. Mitosis in human osteoarthritic cartilage. Clin. Orthop. 1972; 88:247.

3. Ryu, J., Treadwell, B.V. and Mankin, H.J. Biochemical and metabolic abnormalities in normal and osteoarthritic human articular cartilage. Arthritis Rheum. 1984; 27:49.

4. Lippiello, L., Hall, D. and Mankin, H.J. Collagen synthesis in normal and osteoarthritic human cartilage. J. Clin. Invest. 1977; 59:593.

5. Brandt, K.D. Enhanced extractability of articular cartilage protoglycans in osteoarthritis. Biochem. J. 1974; 143:475.

6. Peyron, J.G. and Altman, R.D. The Epidemiology of
Osteoarthritis. In: Osteoarthritis: Diagnosis and Management. 2nd ed, Moskowitz, R.W., Howell, D.S., Goldberg, V.C., Mankin, H.J. (Eds), WB Saunders, Philadelphia 1992. p.15.

7. Martel-Pelletier J., Faure, M.P., McCollum, et al. Plasmin, plasminogen acitvators and inhibitor in human osteoarthritic cartilage. J. Rheumatol. 1991; 18: $1863-1871$.

8. van de Loo F.A., Joosten, L.A., van Lent P.L, Arntz, O.J. and van den Berg W.B., Role of interleukin-1, tumor necrosis factor alpha, and interleukin-6, in cartilage proteoglycan metabolism and destruction. Effect in situ blocking in the murine antigen- and zymogeninduced arthritis. Arthritis Rheum. 1995; 38:164-172.

9. Plows D., Probert L., Georgopoulos S., Alexpoulou L. and Kollias $\mathrm{G}$. The role of tumour necrosis alpha(TNF) in arthritis: studies in transgenic mice. Rheumatol Eur. 1995; Suppl 2: 51-54.

10. Clancy R. Nitrous oxide alters chondrocyte function by disrupting cytoskeleton signaling complexes. Osteoarthritis and Cartilage. 1999; 7:399-400.

11. Schumacher, H.R. Synovial inflammation, crystals and osteoarthritis. J. Rheumatol. 1995; 22(Suppl 43):101.

12. Dumond $\mathrm{H}$, Presle N, Terlain B, et al. Evidence for a key role of leptin in osteoarthritis. Arthritis Rheum. 2003; 48 (11):3118-3129.

13. Bonnet, C. S. and Walsh D. A. Osteoarthritis, angiogenesis and inflammation. Rheumatology.2005; 44: 7-16.

14. Sakkas L.I. and Platsoucas C.D. The role of T cells in the pathogenesis of osteoarthritis. Arthritis Rheum. 2007; 56 (2):409-424.

15. Pelletier J.P., Jovanovic D, Fernandes J.C, et al. Reduced progression of experimental osteoarthritis in vivo by selective inhibition on inducible nitrous oxide synthase. Arthritis Rheum. 1998; 41: 1275-1286.

16. Uebelhart D. Clinical review of chondroitin sulphate in osteoarthritis. Osteoarthritis Cartilage. 2008; 16: S19-21. 